\title{
Una aproximación al estudio de la antroponimia en Honduras durante el periodo colonial (siglos XVII-XVIII)
}

\author{
José Armando San Martín Gómez
}

\section{(9) $(1) \Theta \Theta$}

Doi: https://doi.org/10.15517/rfl.v47i1.44385

URL: https://revistas.ucr.ac.cr/index.php/filyling/index 
Lingüística

Una aproximación al estudio de la antroponimia en Honduras durante el periodo colonial (siglos XVII-XVIII)

\section{An Aproximation to The Study of Honduran Anthroponomy During Colonial Period (17th-18th Centuries)}

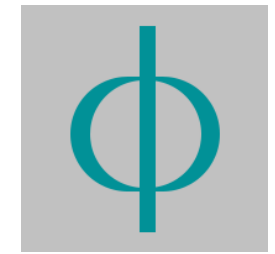

San Martín Gómez, José Armando

José Armando San Martín Gómez armandosmg21@gmail.com

Investigador independiente, Querétaro, México

Revista de Filología y Lingüística de la Universidad de Costa Rica

Universidad de Costa Rica, Costa Rica

ISSN: 0377-628X

ISSN-e: 2215-2628

Periodicidad: Semestral

vol. 47 , núm. 1,2021

filyling@gmail.com

Recepción: 28 Mayo 2020

Aprobación: 17 Junio 2020

URL: http://portal.amelica.org/ameli

jatsRepo/125/1251597034/index.htm

DOI: https://doi.org/10.15517/rfl.v47i1.44385
Resumen: Al igual que ocurre en otras zonas de Centroamérica, la historia de la antroponimia en Honduras es un tema que ha sido descuidado por parte de los investigadores. Precisamente por eso, el siguiente estudio tiene la intención de analizar el comportamiento de los nombres propios en Honduras durante los siglos XVII y XVIII; de este modo, se estudiarán los 33 documentos de esta región editados por Ramírez Luengo (2017). En concreto, los objetivos que pretende alcanzar esta investigación son: a) describir el uso y empleo del nombre propio; b) analizar la distribución de la antroponimia en función de criterios sociales; c) establecer una comparación con otras zonas de dominio hispánico durante estas centurias. La meta final es abrir nuevas líneas de investigación que contribuyan a la historia de la onomástica en Centroamérica.

Palabras clave: antroponimia, Honduras, usos, tendencias, siglos XVII y XVIII.

\begin{abstract}
The history of the anthroponomy of Honduras remains understudied until now, as it happens in other Central American regions. This paper aims to study the forenames used in Honduras during 17th and 18th centuries. For this purpose, a corpus of 33 historical documents (Ramírez Luengo, 2017) will be studied in order to: a) describe the usage of forenames in Honduras; $b$ ) analyze the distribution of the anthroponomy in regard to social criteria; c) compare the usage of forenames in other Hispanic zones during these centuries. The final goal is to increase the knowledge of historical anthroponomy in Honduras and also in Central America.
\end{abstract}

Keywords: anthroponomy, Honduras, uses, trends, 17 th and 18 th centuries.

\section{LA Historia de LA ANTROPONIMIA EN CENTROAMÉRICA: UNA PÁGINA EN BLANCo}

Actualmente la historia de la onomástica, materia que ha llamado la atención de distintas parcelas del saber por ser un objeto de estudio interdisciplinar (Billy, 1993, p. 5; Rebollo Torío, 1995, p. 399; Roldán Sánchez, 1998, p. 273) ${ }^{1}$, "sigue siendo uno de los campos menos roturados de la labor filológica" (Buesa Oliver y Lagüens García, 1996-1997, p. 11), pues si bien la toponimia "ha sido la más trabajada y la que más atención ha merecido a eruditos y estudiosos” (Sánchez Rubio y Testón Núñez, 2010, p. 75), el análisis histórico de los 
nombres de persona ha sido un tópico que ha quedado por mucho tiempo descuidado. Con todo, es posible facilitar en este estado de la cuestión una serie de trabajos realizados en España y en el continente americano sobre este tema para así ubicar mejor el punto de partida y el contexto de este estudio.

Más allá de trabajos que trascienden una zona específica (Becker, 2018; García Gallarín, 2014b; Castro Alfín, 2014), en el caso de España el periodo medieval ha sido analizado en múltiples ocasiones, tanto de manera general (Simón Parra, 2008; García Gallarín, 1998) como para ámbitos geográficos más concretos, por ejemplo Aragón (Buesa Oliver, 1995; Buesa Oliver y Lagüens García, 1996-1997) y Sevilla (Rodríguez Toro, 2010); para periodos posteriores, se cuenta con el estudio de Carrasco García (2008-2010) sobre los moriscos granadinos del siglo XVI, Galende Díaz y García Gallarín (2006), García Gallarín (2019) sobre este mismo siglo o Zabalza Seguín (2014) para toda la época moderna, entre 1570 y 1870.

Frente a la situación de España, donde el estudio de la historia onomástica ha experimentado un gran interés, lo cierto es que América no ha tenido este privilegio, de modo que las investigaciones realizadas en este continente son escasas. A este respecto, se puede mencionar las aportaciones de García Gallarín (2007a, 2010), así como trabajos más específicos que hablan de cuestiones muy puntuales, como la influencia de la religión y el culto de los santos en el nombre de persona (Zabalza Seguín, 2008) ${ }^{2}$ u otros que analizan regiones como México (Boyd-Bowman, 1970; Brambila Paz y Villegas Molina, 2020; López Franco, 2020; Reyes Contreras, 2020), Argentina, Costa Rica o El Salvador (Díaz de Martínez, 2003; Quesada Vargas, 2010; San Martín Gómez, s. f.). Otras áreas del continente, sin embargo, no han sido todavía analizadas, y a este respecto la historia de los nombres propios en Honduras es hasta ahora totalmente desconocida, de manera que se hace necesario prestar atención a este país centroamericano para así ofrecer una visión más amplia del conjunto de procesos que han determinado la configuración antroponímica de este territorio ${ }^{3}$.

\section{OBJETIVOS Y CORPUS DE TRABAJO}

De este modo, y teniendo en cuenta el estado de la cuestión, el presente trabajo tiene la intención de brindar información sobre la historia del nombre propio en América Central -muy específicamente en Honduras- a partir del estudio de la documentación inquisitorial de los siglos XVII y XVIII editada en Ramírez Luengo (2017). En concreto, los objetivos que pretende alcanzar esta investigación son: a) conocer el uso que presenta el nombre de pila en el español de Honduras durante estas dos centurias, mediante el análisis de cuestiones como los nombres empleados, la cantidad de ellos, su frecuencia de uso, la presencia de estructuras de nombre simple y compuesto, etc.; b) analizar la distribución de los nombres propios para descubrir si existe alguna preferencia de acuerdo con criterios sociolingüísticos; c) en la medida de lo posible, comparar la antroponimia de Honduras, El Salvador y otras zonas del dominio hispánico entre 1650 y 1819 para establecer las similitudes y las diferencias existentes entre ellas; y d) a partir de este análisis, abrir nuevas líneas de investigación que contribuyan al enriquecimiento de la historia de la onomástica en Centroamérica y América en general.

Por lo que se refiere al corpus de trabajo, se ha optado por utilizar 33 documentos notariales de esta región (Ramírez Luengo, 2017) ${ }^{4}$ que proceden de los fondos inquisitoriales del Archivo General de la Nación (AGN; Ciudad de México, México); respecto a su ámbito geográfico y temporal, estos se encuentran repartidos entre 1650 y 1819 y provienen de todo el país, específicamente de Choluteca, Comayagua, Curaren, Ojojona, Olanchito, Olancho, Olancho el Viejo, Omoa, Tegucigalpa, Trujillo y Yoro. En cuanto a su tipología textual, se encuentran con mayor frecuencia algunos tipos específicos como por ejemplo denuncias, interrogatorios y testimonios, aunque también se incorporan otros como inventarios de bienes y cartas privadas; hay que tener en cuenta que la naturaleza inquisitorial de estos documentos hace que muchos de ellos, por sus características, se aproximen al polo de la inmediatez comunicativa (Oesterreicher, 2004, p. 752). 


\section{ANÁlisis de los dATOS: la ANTROponimia EN la HoNDURAS TARDocolonial}

En lo que respecta a los nombres extraídos de la documentación, es necesario señalar primeramente que aparece un total de 172 casos que se reparten en 66 nombres diferentes: en concreto, se pueden apreciar en el primer periodo (1650-1700) 74 apariciones y 38 nombres distintos, mientras que la primera mitad del siglo XVIII cuenta con un total de 13 ejemplos y 12 designaciones y la segunda mitad de este siglo aporta un total de 85 y 56 respectivamente 5 . Por tanto, parece interesante realizar un análisis de la variedad de nombres empleados en cada corte cronológico, y para este propósito es necesario interpretar estos resultados a través del indice de variación onomástica (IVO), que se entiende como "la distribución de nombres diferentes asignados a la población de un corpus de estudio” (San Martín Gómez, s. f. ${ }^{6}$. Así, a partir de los antropónimos extraídos de los textos estudiados, se han obtenido los resultados siguientes (Tabla 1):

TABLA 1.

Distribución total de los nombres propios del siglo XVII y XVIII en el corpus

\begin{tabular}{|l|l|l|l|}
\hline Periodo analizado & Nombres distintos & Total de nombres & IVO \\
\hline $1650-1700$ & 38 & 74 & 0.51 \\
\hline $1701-1750$ & 12 & 13 & 0.92 \\
\hline $1751-1819$ & 56 & 85 & 0.66 \\
\hline TOTAL & 66 & 172 & 0.38 \\
\hline
\end{tabular}

Por consiguiente, la aplicación de este índice de variación onomástica permite descubrir la existencia de un cambio cronológico durante las tres épocas en las que se ha segmentado la documentación: en efecto, aunque parece existir un aumento discontinuo -al pasar del 0.51 al 0.92 y descender durante la segunda mitad del siglo $\mathrm{XVIII}^{7}$ a un 0.66 -, hay que tener en cuenta que los resultados obtenidos de la primera mitad del siglo XVIII, tomados de un único documento, son relativamente escasos y pueden desvirtuar la progresión real, de manera que parece posible sostener la hipótesis de un crecimiento constante del IVO -es decir, una mayor variación de nombres en la comunidad- entre los siglos XVII y XVIII, algo que también se puede apreciar en las investigaciones desarrolladas sobre España (Zabalza Seguín, 2008, pp. 109-118) y El Salvador (San Martín Gómez, s. f.).

Más allá de esta primera aproximación general, y pasando ahora de manera más puntual a un análisis por sexo, resulta interesante también llevar a cabo un estudio semejante al anterior para poder observar así con mayor precisión el comportamiento del nombre propio hondureño de acuerdo con esta variable. En concreto, la información obtenida es la que se presenta a continuación (Tabla 2$)^{8}$ : 
TABLA 2.

Distribución del nombre propio (masculino/femenino) durante los siglos XVII y XVIII

\begin{tabular}{|l|l|l|}
\hline Periodo analizado & Nombres masculinos & Nombres femeninos \\
\hline $1650-1700$ & $33 / 63(0.52)$ & $5 / 11(0.45)$ \\
\hline $1701-1750$ & $9 / 11(0.82)$ & $2 / 2(1)$ \\
\hline $1750-1819$ & $43 / 72(0.60)$ & $13 / 13(1)$ \\
\hline TOTAL & $66 / 146(0.45)$ & $16 / 26(0.61)$ \\
\hline
\end{tabular}

De este modo, se puede observar que durante el periodo $1650-1700$ el nombre de pila masculino 9 muestra un valor de 0.52 en el índice de variación onomástica que pasa al 0.82 en la primera mitad del siglo XVIII y desciende al 0.60 durante el periodo de 1750-1819. Pasando ahora al caso del nombre de pila femenino, es interesante observar que la tendencia al aumento también se produce, pues si durante el siglo XVII el IVO corresponde a 0.45 , en el siglo XVIII aumenta y se mantiene hasta su máximo grado (1). Así pues, los resultados dejan ver que ambos sexos tienen un comportamiento similar en lo que se refiere al aumento constante del IVO, si bien se detecta una diferencia de grado: mientras que en los hombres la variación de nombres es menor, en el caso de las mujeres se registra una mayor cantidad de nombres diferenciados, al alcanzar el valor de 1 , el máximo posible.

Por lo que se refiere a la estructura de los antropónimos analizados, este repositorio documental ofrece casos tanto de nombres simples como de nombres compuestos, que en palabras de Reyes Díaz y Marrero Pulido (2013, pp. 192-193) estos últimos suponen "la unión de dos nombres que, mediante la yuxtaposición [...] conforman otra entidad distinta constituida por dos elementos léxicos" ${ }^{\prime 1}$. En concreto, se ha registrado un total de 133 nombres simples y 39 compuestos, el $77.3 \%$ y el $22.7 \%$ respectivamente, lo cual demuestra la superioridad cuantitativa de los primeros, así como cierta evolución a lo largo de los tres periodos estudiados. Los resultados de este análisis se pueden apreciar en la siguiente tabla (Tabla 3):

TABLA 3.

Distribución de la estructura de antropónimos estudiados en el corpus

\begin{tabular}{|l|l|l|}
\hline Periodo analizado & Simple & Compuesto \\
\hline $1650-1700$ & $69(93.24 \%)$ & $5(6.76 \%)$ \\
\hline $1701-1750$ & $10(77 \%)$ & $3(23 \%)$ \\
\hline $1751-1819$ & $54(63.5 \%)$ & $31(36.5 \%)$ \\
\hline TOTAL & $133(77.3 \%)$ & $39(22.7 \%)$ \\
\hline
\end{tabular}

En cuanto a la comparación de las tres épocas, es necesario hacer hincapié en el proceso de cambio porcentual que tiene el nombre compuesto frente al simple, pues la frecuencia del primero avanza progresivamente de forma significativa: efectivamente, la preponderancia casi absoluta del nombre simple 
-en concreto 69 nombres, correspondientes al $93.24 \%$ - se va reduciendo a lo largo del tiempo a favor de las estructuras compuestas, pasando así al 77 \% (1701 y 1750) y al $63.5 \%$ durante la segunda mitad de este siglo, lo que supone un aumento del nombre compuesto de alrededor de un $15 \%$ en cada época estudiada ${ }^{11}$, en una situación que repite de manera muy semejante lo que, a este respecto, se apuntó ya para el español salvadoreño (San Martín Gómez, s. f.).

Más allá de la descripción general, teniendo en cuenta ahora una clasificación por sexo, es interesante asimismo observar su estructura a lo largo del tiempo, para así comprobar si el proceso de expansión del nombre compuesto afecta igualmente a ambos sexos. En lo que se refiere a su análisis cuantitativo, se registra un total de 22 nombres simples y 4 compuestos en las mujeres, es decir, el $84.61 \%$ y el $15.39 \%$ respectivamente, mientras que en el caso de los hombres se descubren 113 elementos simples y 33 compuestos -con porcentajes del $77.4 \%$ y del $22.6 \%$ respectivamente-, cuya distribución cronológica se aprecia en la siguiente tabla $(\text { Tabla } 4)^{12}$ :

TABLA 4.

Distribución de antropónimos simples y compuestos en el corpus

\begin{tabular}{|c|c|c|c|c|}
\hline \multirow{2}{*}{$\begin{array}{l}\text { Periodo } \\
\text { analizado }\end{array}$} & \multicolumn{2}{|c|}{ Femenino } & \multicolumn{2}{|c|}{ Masculino } \\
\hline & Simple & Compuesto & Simple & Compuesto \\
\hline $1650-1700$ & $11(100 \%)$ & $0(0 \%)$ & $59(93.65 \%)$ & $4(6.35 \%)$ \\
\hline $1701-1750$ & $2(100 \%)$ & $0(0 \%)$ & $9(81.81 \%)$ & $2(18.19 \%)$ \\
\hline $1751-1803$ & $9(69.2 \%)$ & $4(30.8 \%)$ & $45(62.5 \%)$ & $27(37.5 \%)$ \\
\hline TOTAL & $22(84.61 \%)$ & $4(15.39 \%)$ & $113(77.4 \%)$ & $33(22.6 \%)$ \\
\hline
\end{tabular}

Así pues, a pesar de la escasez de casos femeninos, los porcentajes extraídos parecen esbozar un proceso paralelo en ambos grupos, lo que pone de manifiesto que el aumento de la frecuencia de uso del nombre compuesto no depende ni es característico de un sexo en particular, sino que este cambio de tendencia se produce y se expande más bien de forma general, una vez más a semejanza de lo que ocurre en El Salvador, donde se presenta el mismo comportamiento (San Martín Gómez, s. f.) ${ }^{13}$.

En cuanto a los nombres concretos en sí, el estudio de la documentación permite dar cuenta de las preferencias que existen en los distintos cortes cronológicos al momento de seleccionar un nombre de pila en el territorio hondureño, muy específicamente en lo que concierne al caso de los nombres simples ${ }^{14}$, todo lo cual permite observar cómo ha sido la evolución de la configuración antroponímica en este país. De este modo, comenzando así por la antroponimia masculina, los resultados que se registran en los documentos son los siguientes $(\text { Tabla } 5)^{15}$ : 
TABLA 5.

Apariciones del nombre masculino simple en el corpus

\begin{tabular}{|c|c|c|c|}
\hline Apariciones & $1650-1700$ & $1701-1750$ & 1751-1819 \\
\hline 10 casos & & & Francisco \\
\hline 8 casos & Francisco, Juan & & \\
\hline 4 casos & Alonso, Antonio & & \\
\hline 3 casos & $\begin{array}{ll}\text { José, } & \text { Pedro, } \\
\text { Sebastián } & \end{array}$ & & $\begin{array}{l}\text { Antonio, José, } \\
\text { Miguel }\end{array}$ \\
\hline 2 casos & $\begin{array}{l}\text { Cristóbal, Luis, } \\
\text { Manuel }\end{array}$ & Cipriano, Diego & $\begin{array}{lr}\text { Bernardo, } & \text { Juan, } \\
\text { Joaquín, } & \text { Manuel, } \\
\text { Mariano, } & \text { Pedro, } \\
\text { Tomás } & \end{array}$ \\
\hline 1 caso & $\begin{array}{l}\text { Baltasar, } \\
\text { Bernardino, Fabián, } \\
\text { Felipe, Fernando, } \\
\text { Gabriel, } \quad \text { Gaspar, } \\
\text { Gregorio, Isidro, } \\
\text { Lucas, Marcos, } \\
\text { Martín, Mateo, } \\
\text { Matías, Melchor, } \\
\text { Nicolás, Pablo, } \\
\text { Rodrigo, Tomás }\end{array}$ & $\begin{array}{l}\text { Antonio, Claudio, } \\
\text { Cristóbal, José }\end{array}$ & $\begin{array}{lr}\text { Elias, } & \text { Felipe, } \\
\text { Joaquín, } & \text { Laureano, } \\
\text { Luis, } & \text { Marcelino, } \\
\text { Matias, } & \text { Nicolás, } \\
\text { Rafael, } & \text { Ramón, } \\
\text { Santiago, } & \text { Tiburcio, } \\
\text { Ventura } & \end{array}$ \\
\hline
\end{tabular}

Como se puede apreciar, durante la segunda mitad del siglo XVII se observa un claro predominio en Honduras de nombres de populares santos católicos como Francisco, Juan ${ }^{16}$, Antonio, Pedro, José, y Sebastián, seguidos de otros más esporádicos como Cristóbal, Luis, Manuel, Baltasar, Bernardino, Fabián, Felipe, Fernando, Gabriel, Gaspar, Gregorio, Isidro, Lucas, Marcos, Martín, Mateo, Matias, Melchor, Nicolás, Pablo, Rodrigo, y Tomás, en una situación que cambia un poco entre 1701 y 1750, donde los nombres que más predominan son Cipriano y Diego; por último, durante la segunda mitad del siglo XVIII el nombre simple más destacado es Francisco seguido por otros de carácter religioso como Antonio, José, Miguel, Bernardo, Juan, Joaquin, Manuel, Mariano, Pedro, Tomás y otros más esporádicos como Elías, Felipe, Joaquin, Laureano, Luis, Marcelino, Matías, Nicolás, Rafael, Ramón, Santiago, Tiburcio y Ventura.

En lo que se refiere a las mujeres, pese a que la información es mucho más escasa, y, por tanto, no es posible llegar a conclusiones definitivas, parece descubrirse la existencia de una situación semejante a la del nombre de pila masculino, pues se observa que hay una clara preferencia, nuevamente, por el uso de hagiónimos ${ }^{17}$ de gran devoción popular; a continuación se observa en la tabla los nombres extraídos de la documentación analizada (Tabla 6): 
TABLA 6.

Frecuencia de uso del nombre femenino simple en el corpus

\begin{tabular}{|c|c|c|c|}
\hline Apariciones & $1650-1700$ & $1701-1750$ & $1751-1819$ \\
\hline 4 casos & María & & \\
\hline 3 casos & Isabel & & \\
\hline 2 casos & Juana & & \\
\hline 1 caso & Nicolasa, Petrona & Antonia, Gertrudis & $\begin{array}{l}\text { Antonia, Catarina, } \\
\text { Dionisia, Isabel, } \\
\text { Isidora, Jerónima, } \\
\text { Joaquina, Juana, } \\
\text { Teresa }\end{array}$ \\
\hline
\end{tabular}

Así, la tabla pone de manifiesto la presencia a lo largo de los tres períodos de nombres relacionados con santos populares (María, Isabel), muchas veces la versión femenina de los masculinos mayoritarios (Juana y Antonia), que se acompañan de otros ocasionales como Nicolasa, Petrona, Gertrudis, Catarina, Dionisia, Isidora, Jerónima, Joaquina, y Teresa. A este respecto, parece relevante señalar que estos datos coinciden con los de México, donde, junto a Josepha, Manuela, Gertrudis o Rosa, se descubre un uso frecuentísimo de María (Boyd-Bowman, 1970, pp. 27-28), a semejanza de lo que ocurre en estos documentos y en otras zonas como del dominio hispánico como la misma España (Zabalza Seguín, 2008, pp. 115-117); por el contrario, esta situación difiere con El Salvador, pues durante estas centurias no hay ninguna aparición de este nombre.

Pasando al último análisis, que trata de la relación entre la clase social y la onomástica ${ }^{18}$, la división de los individuos de los materiales analizados en dos clases -alta y baja- según su ocupación permite detectar si existe alguna relación entre esta circunstancia y el uso del nombre simple o compuesto ${ }^{19}$. En concreto, los datos que se extraen de la documentación hondureña analizada son los siguientes (Tabla 7): 
TABLA 7.

Distribución de la onomástica simple y compuesta por clase social en el corpus

\begin{tabular}{|l|l|l|l|l|}
\hline \multirow{2}{*}{ PERIODO } & \multicolumn{2}{|c|}{ CLASE ALTA } & \multicolumn{2}{c|}{ CLASE BAJA } \\
\cline { 2 - 5 } & Simple & Compuesto & Simple & Compuesto \\
\hline $1650-1700$ & $38(90.47 \%)$ & $4(9.53 \%)$ & $3(100 \%)$ & $0(0 \%)$ \\
\hline $1700-1750$ & $8(80 \%)$ & $2(20 \%)$ & $1(100 \%)$ & $0(0 \%)$ \\
\hline $1750-1803$ & $26(59.1 \%)$ & $18(40.9 \%)$ & $3(100 \%)$ & $0(0 \%)$ \\
\hline TOTAL & $72(75 \%)$ & $24(25 \%)$ & $7(100 \%)$ & $0(0 \%)$ \\
\hline
\end{tabular}

Así pues, los resultados de la tabla demuestran que, en el caso de la clase alta, mientras que en el período 1650-1700 aparece un claro predominio del nombre simple, esta situación va variando a lo largo del siglo XVIII con un aumento progresivo del compuesto, en una situación que no se da en la clase baja, que mantiene en todas las ocasiones y a lo largo del tiempo el uso de la forma simple. A partir de estos datos, y a pesar de su escasez, parece posible mantener la hipótesis de que, si bien en el siglo XVII el empleo del nombre simple y compuesto no muestra un reparto de acuerdo con la clase social, en apenas cien años esta estructura se ha convertido en un marcador social que identifica exclusivamente a los grupos elevados de la población en Honduras, algo que también se descubre, y de manera más evidente, en el caso salvadoreño (San Martín Gómez, s. f. $)^{20}$.

\section{Conclusiones}

Teniendo en cuenta las informaciones extraídas de estos documentos y los análisis llevado a cabo, es posible aportar una breve serie de conclusiones que arrojan luz sobre la historia de la onomástica personal en Centroamérica, y muy específicamente en Honduras; de este modo, a partir de las líneas escritas anteriormente, los resultados obtenidos y las comparaciones hechas con otros países, tales conclusiones son las siguientes.

En primer lugar, y en lo que respecta a la variación onomástica en Honduras durante el periodo tardocolonial, cabe decir que el indice de variación onomástica (IVO) deja ver un enriquecimiento en la cantidad de apelativos utilizados en la región cuando se observa que este inicia con un 0.51 durante la segunda mitad del siglo XVII y termina con un 0.66 en el periodo de la última centuria. Asimismo, el aumento del IVO para ambos sexos muestra similitudes, por lo que se hace necesario interrogarse si el comportamiento de los antropónimos en la documentación se debe a las nuevas modas del momento histórico o si responde más bien a cuestiones de otra naturaleza, en lo que sin duda constituye una línea de trabajo que necesitará desarrollarse con mayor profundidad en el futuro.

En cuanto al empleo de la estructura antroponímica, se descubre que en los documentos analizados la frecuencia de uso del nombre compuesto avanza progresivamente de forma muy significativa, al pasar del $5.4 \%$ al $36.5 \%$ del total, en un proceso que no parece estar determinado por el sexo; cabe decir que este hecho no sorprende, pues el estudio de este tema en España y El Salvador (Zabalza Seguín, 2008, pp. 118-119; San Martín Gómez, s. f.) demuestra el mismo comportamiento de Honduras, si bien ambos países centroamericanos parecen seguir las mismas tendencias de la Península con un cierto atraso respecto a esta. 
Por lo que se refiere a los nombres más comunes del corpus, se observa en la Honduras tardocolonial un claro predominio de hagiónimos en ambos sexos, tales como Francisco, Juan, Antonio, Pedro, José, Sebastián para el caso masculino y María, Isabel, Juana, Antonia para el femenino. En este punto concreto, es interesante observar que la marcada preferencia por María coincide con la que se da en otras regiones como España en el siglo XVI (Zabalza Seguín, 2008) y México en los siglos XVII y XVIII (Boyd-Bowman, 1970), si bien contrasta con lo que se registra en otras regiones como El Salvador (San Martín Gómez, s. f.).

Por otro lado, los resultados presentes del corpus permiten también estudiar la relación entre la clase social del sujeto y el empleo del nombre simple o compuesto, tema que hasta el momento ha sido poco estudiado. En concreto, a partir de estos análisis es posible llegar a dos conclusiones interesantes: en primer lugar, que el nombre compuesto no es una marca social durante el siglo XVII, dado que ambas clases lo utilizan de forma muy minoritaria y con porcentajes muy cercanos; en segundo lugar, que esta situación cambia a lo largo del siglo XVIII, pues en su segunda mitad, mientras que el nombre simple sigue predominando en la clase baja, la clase alta presenta apenas un $60 \%$ de este frente a un $40 \%$ del compuesto.

Finalmente, tras el análisis hecho, parece necesario cerrar estas páginas haciendo hincapié en una serie de cuestiones que pueden contribuir a desarrollar el estudio de esta cuestión: a manera de ejemplo, es necesario prestar atención a otras cronologías no atendidas como por ejemplo el siglo XIX, para así poder determinar si estas tendencias tienen continuidad en el tiempo a pesar de los cambios sociohistóricos que tienen lugar en la época; asimismo, hace falta analizar también otras regiones del continente, para ver si existen coincidencias entre las distintas áreas geográficas; por último, también se debe describir de manera más profunda la situación de la onomástica personal en la misma Honduras tardocolonial, cuidando aspectos como pueden ser las zonas dialectales del país, la oposición ciudad/campo o la comparación de los nombres de las diferentes $\operatorname{castas}^{21}$, todo lo cual enriquecerá nuestro conocimiento de la historia del nombre propio en este país, así como en América de manera general.

\section{Bibliografía}

Becker, L. (2018). Nombres de persona en español. Historia, situación actual y onomástica popular. Berlín: Peter Lang. Billy, P. (1993). Le nom propre et le nom sale. Nouvelle Revue d'Onomastique, (21-22), 3-10.

Boyd-Bowman, P. (1970). Los nombres de pila en México desde 1540 hasta 1950. Nueva Revista de Filología Hispánica (NRFH), 19(1), 12-48.

Brambila Paz, R. y Villegas Molina, M. E. (2020). Antroponimia registrada en las Mercedes de Jilotepec, siglo XVI. Onomástica Desde América Latina, 1(1), 118-140.

Buesa Oliver, T. (1995). De onomástica en Aragón. Archivo de Filología Aragonesa, 51, 269 - 281.

Buesa Oliver, T. y Lagüens García, V. (1995). Apellidos con artículo en Aragón referidos a edificios y otras construcciones. Thesaurus: Boletin del instituto Caro y Cuervo, 50(1-3), 239-292.

Buesa Oliver, T. y Lagüéns Gracia, V. (1996). Algunos apellidos con artículo en Aragón. En A. Alonso González (Coord.), Actas del III Congreso Internacional de Historia de la Lengua Española (Salamanca, 22-27 de noviembre de 1993) (pp. 981-1005). Madrid: AHLE, Arco/Libros y Fundación Duques de Soria.

Buesa Oliver, T. y Lagüens García, V. (1996-1997). Trabajos del centro "PatRom" de Zaragoza (I). Los estudios de la antroponimia aragonesa. Archivo de filologia aragonesa, (52-53), 9-64.

Cabrera Pombrol, P. (2006). Notas históricas y genealógicas del apellido "Pedrianes" en la Palma. Revista de estudios generales de la isla de la isla de La Palma, 2, 31-44.

Carrasco García, G. (2008-2010). La onomástica de la conversión: señas de identidad y transformación antroponímica de los moriscos de Granada (1500-1569). Sharq Al-Andalus, 19, 139-204.

Castro Alfín, D. (2014). Antroponimia y sociedad. Una aproximación sociobistórica al nombre de persona como fenómeno cultural. Pamplona: Universidad Pública de Navarra. 
Díaz de Martínez, L. (2003). Onomástica mariana en las actas de bautismo de Humahuaca (Jujuy-Argentina) del siglo XVIII. En Lexicografia y lexicología en Europa y América: Homenaje a Günter Haensch (pp. 239-248). Madrid: Gredos.

Elián, J. S. (2001). El gran libro de los apellidos y la heráldica. Barcelona: Ediciones Rainbook.

Galende Díaz, J. C. y García Gallarín, C. (2006). Onomástica y deonomástica del siglo XVII. En V Jornadas Cientificas sobre Documentación en España e Indias durante el siglo XVII. Madrid: Universidad Complutense.

García Gallarín, C. (1988). El nombre propio de la persona. Marca social en la literatura española del siglo XVII. Actas del I Congreso Internacional de Historia de la Lengua Española, 2, 1707-1716.

García Gallarín, C. (1998). Los nombres de pila españoles. Madrid: Ediciones del Prado.

García Gallarín, C. (2001). Los gentilicios recategorizados en apellidos: Contribución al estudio de la influencia norteña en el Madrid de 1600 a 1630. Rion, 2, 443-458.

García Gallarín, C. (2007a). La evolución de la antroponimia hispanoamericana. En C. García Gallarín (Coord.), Los nombres del Madrid multicultural (pp. 209-235). Madrid: Parthenon.

García Gallarín, C. (2007b). Tradición e innovación antroponímicas (Madrid, 1996-2006). En C. García Gallarín (Coord.), Los nombres del Madrid multicultural (pp. 99-134). Madrid: Parthenon.

García Gallarín, C. (2010). Rutas de la antroponimia hispánica. En C. Maíz (Coord.). Nombre propio e identidad (pp. 59-99). Madrid: Silex Ediciones.

García Gallarín, C. (2014a). Nombre propio y ambigüedad. En J. J. Ortega y M. V. Navas (Coords.), Nombre oficial y nombre familiar (pp. 43-73). Madrid: Ediciones del Orto.

García Gallarín, C. (2014b). Diccionario histórico de nombres de América y España. Estudio Preliminar. Madrid: Silex Ediciones.

García Gallarín, C. (2019). Antroponimia teresiana. En L. Albuquerque García, J. L. García Barrientos, A. Garrido Domínguez y A. Suárez Miramón (Coords.), Vir bonus dicendiperitus: homenaje al profesor Miguel Ángel Garrido Gallardo (pp. 195-207). Madrid: Consejo Superior de Investigaciones Científicas.

López Franco, Y. G. (2020). Las relaciones intercategoriales e intracategoriales en antroponimia. El caso de los nombres de pila en francés de Francia y en español de México. Onomástica Desde América Latina, 1(1), 214-239.

Mendoza Abreu, J. M. (2009). Estudio histórico de apellidos andaluces medievales. Madrid: Arco Libros.

Oesterreicher, W. (2004). Textos entre inmediatez y distancia comunicativas. El problema de lo hablado escrito en el Siglo de Oro. En R. Cano Aguilar (Coord.), Historia de la Lengua Española (pp. 729-769). Barcelona: Ariel.

Quesada Vargas, M. (2010). Onomástica juaviñense. Kañina, 34(1), 129-145.

Ramírez Luengo, J. L. (2017). Textos para la historia del español XI. Honduras y El Salvador. Alcalá de Henares: Universidad de Alcalá.

Reyes Contreras, M. (2020). Truncamientos de hipocorísticos españoles y nombres de pila en mazahua: un fenómeno compartido. Onomástica Desde América Latina, 1(1), 141-167.

Reyes Díaz, M. J. y Marrero Pulido, V. (2013). Antropónimos. Análisis de una muestra canaria de los últimos dos decenios. Nouvelle revue d'onomastique, (55), 191-231.

Rebollo Torío, M. Á. (1995). El nombre propio y su significado. Anuario de estudios filológicos, (18), 399-406.

Rodríguez Toro, J. J. (2010). Los nombres de pila españoles en época preclásica (según el padrón general de Sevilla, año 1533). Nouvelle revue d'onomastique, (52), 221-235.

Roldán Sánchez, R. (1998). Bases metodológicas para un estudio sobre la onomástica contemporánea. Interlingüistica, 9, 273-276.

Saavedra, P. (1994). La vida cotidiana en la Galicia del Antiguo Régimen. Barcelona: Crítica.

Sánchez Rubio, M. R. y Testón Núñez, I. (2010). Situación y perspectiva de los estudios de antroponimia en la España Moderna. En A. Addobbati, R. Bizzocchi y G. Salinero (Coords.), L'Italia dei cognomi: l'antroponimia italiana nel quadro mediterraneo (pp. 75-122). Pisa: University Press. 
San Martín Gómez, J. A. (s. f.). "Estudio de la antroponimia de El Salvador tardocolonial (1650-1803): tendencias de uso y evolución” (inédito).

Simón Parra, M. (2008). El nombre depersona en la documentación castellana medieval (Tesis doctoral inédita). Alcalá de Henares: Universidad de Alcalá.

Vallejo Ruiz, J. M. (2009). La validez de la antroponimia como fuente de estudio de las lenguas antiguas: El caso de la Península Ibérica. Emerita, 77(1), 125-145.

Zabalza Seguín, A. (2008). Nombres viejos y nombres nuevos. Sobre la onomástica moderna. Memoria y Civilización, (11), 105-134.

Zabalza Seguín, A. (2014). Del Concilio de Trento al Registro Civil (1570-1870): la evolución de la onomástica en Navarra. En J. Tort i Donada y M. Montagut i Montagut (Coords.), Els noms en la vida quotidiana: Actes del XXIV Congrès Internacional d'ICOS sobre Ciènces Onomàstiques (pp. 1757-1768). Cataluña: Generalitit de Catalunya.

Zabalza Seguín, A. (2020). Del solar bajonavarro a la Nueva España: el viaje de Juan Jaso (1523). Onomástica Desde América Latina, 1(1), 3-20.

Zurera Álvarez, F. J. (2012). Estudio de un apellido a través de padrones de habitantes en Aguilar de la Frontera durante los siglos XIX y XX. Trastámara, revista de Ciencias Auxiliares de la Historia, 10, 107-119.

\section{Notas}

1 En cuanto a la trascendencia de los nombres propios, se sabe que estos cambian en forma de auténtica moda o costumbre en una sociedad, y algunas veces se fundamentan en motivaciones que van mucho más lejos de las cuestiones lingüísticas (García Gallarín, 2007b, p. 99; Galende Díaz y García Gallarín, 2006, p. 169); esta variación se detecta de generación en generación y, muchas veces, de región en región, y se vuelve aún mucho más evidente cuando se compara en la línea de tiempo la onomástica de un solo lugar (Boyd-Bowman, 1970, p. 12; Reyes Díaz y Marrero Pulido, 2013, p. 204).

2 A este respecto, cabe decir que la onomástica es un reflejo de las devociones de cada momento histórico (Saavedra, 1994, p. 323), lo que permite apreciar cómo los nombres se imponen a lo largo del tiempo por causas tanto históricas como culturales (Vallejo Ruiz, 2009, p. 127).

3 Aunque este trabajo se centra en el nombre propio y no en el apellido, es interesante citar algunas aportaciones dedicadas a este tema, entre las que destacan Buesa Oliver y Lagüens García (1995, 1996), García Gallarín (2001), Elián (2001), Cabrera Pombrol (2006), Mendoza Abreu (2009), y Zurera Álvarez (2012) y Zabalza Seguín (2020).

4 El empleo de documentos en trabajos de esta naturaleza es muy útil, de hecho, y en palabras de Roldán Sánchez (1998, p. 273) "para un estudio de este tipo es casi imprescindible el manejo de fuentes documentales".

5 El resultado final de los nombres distintos no supone la suma de los tres períodos, dado que hay algunos que se repiten en todos los periodos, sino más bien los antropónimos diferentes sobre el total de nombres extraídos de todo el corpus.

6 Algunos autores como Reyes Díaz y Marrero Pulido (2013, p. 192) hacen uso de este concepto en el estudio de los antropónimos del momento actual, aunque no ofrecen ninguna denominación en concreto para él.

7 En lo que respecta a este periodo, aparecen dos documentos entre 1800 y 1819 que mantienen similitudes con la lengua de la segunda mitad del siglo XVIII, por esta razón se ubican dentro de la periodicidad de 50 años y no de 70 como pudiera parecer.

8 Para la lectura de esta tabla, téngase en cuenta que el primer elemento que aparece del lado izquierdo (33) corresponde a los nombres diferentes de la segunda mitad del siglo XVII, los cuales se han dividido entre el total de nombres presentes en el periodo analizado (63); la relación entre ambos genera el indice de variación onomástica, ubicado entre paréntesis $(0.52)$.

9 Cabe señalar que la mayor cantidad de nombres recolectados en el corpus es de naturaleza masculina, en concreto el $84.88 \%$, algo que determina que las conclusiones acerca de este sexo sean mucho más precisas.

10 En este punto en concreto, hay que contar ambas estructuras por separado, algo que sostiene Roldán Sánchez (1998, p. 275) cuando dice que "Juan Antonio sería una denominación distinta de Juan". Sirva como ejemplos de tales nombres compuestos algunos extraídos del corpus (Ramírez Luengo, 2017): para el caso masculino, Félix Antonio Soto (Doc. 24, 1790), Francisco Antonio de Nájera (Doc. 18, 1773) o Félix Antonio de Zuñiga (Doc. 14, 1693); en lo que concierne al nombre femenino, Ana Josefa Galeas (Doc. 31, 1799), Ana María Canales (Doc, 32, 1800) o María del Carmen Lagos (Doc. 32, 1800). 
11 Respecto a este hecho, España parece presentar una popularidad mucho más grande del nombre compuesto, que crece a partir del siglo XVI (Zabalza Seguín, 2008, pp. 118-119), mientras que en América esta moda se presenta un siglo más tarde, al menos en Honduras, El Salvador (San Martín Gómez, s. f.) y México (Boyd-Bowman, 1970, p. 21).

12 Como anteriormente se ha dicho, la falta de datos de la segunda mitad del siglo XVII y la primera del XVIII en cuanto al nombre de pila femenino no permite llegar a conclusiones definitivas en lo que se refiere a la estructura onomástica de este sexo, cuestión que será necesario seguir trabajando en futuras investigaciones.

13 Por lo que se refiere a la estructura de nombres en El Salvador, el caso de la onomástica femenina aumenta esta tendencia de un $18 \%$ en la segunda mitad del siglo XVII a un $40 \%$ en el siglo XVIII; en cuanto a los nombres masculinos, estos pasan del $12 \%$ al $31 \%$ entre 1650 y 1800.

14 Cabe indicar que para este análisis no se han tomado en cuenta los nombres de pila compuestos debido a que la cantidad de ellos es bastante baja.

15 En lo que respecta a las grafías de algunos nombres, se ha optado por adaptarlos a su forma moderna: por ejemplo, Alonzo, Cristóval, Favián, Joseb, Jóan, Joaquín, Marselino, Lauriano, Rodrígo, Sirpiano, Sirbián se transcriben como Cristóbal, Fabián, José, Juan, Joaquín, Laureano, Marcelino, Rodrigo y Cipriano. En cuanto a este particular, García Gallarín (2014a, p. 48) dice que "tanto en las fuentes históricas como en las contemporáneas se encuentran numerosas variantes del mismo nombre propio; algunas representan diferentes fases de un proceso de cambio fónico, otras son testimonio de distintas vías de transmisión".

16 La situación de este nombre es semejante a la de España, en donde tiene una alta popularidad a finales del siglo XVII (Zabalza Seguín, 2008, p. 116).

17 A este respecto, resulta interesante tener en cuenta la observación de García Gallarín (2014b, p. 27) de que "Carlos $\mathrm{V}$ dispuso en 1535 que fueran los misioneros, y no los encomenderos, los encargados de cristianizar y castellanizar; la evangelización conllevaba el cambio de nombre indígena por un hagiónimo", algo que puede explicar la mayor divulgación y popularización de algunos nombres concretos frente a otros.

18 Esta cuestión ha sido poco atendida hasta el momento, pues solo se cuenta con el trabajo de García Gallarín (1988), que hace una aproximación al estudio social de la onomástica a partir de la literatura del siglo XVII. Una vez más, es necesario señalar que este análisis se hará solo con los nombres masculinos.

19 En este sentido, se ha tomado en cuenta el trabajo realizado sobre El Salvador como punto de referencia para determinar como miembros de la clase alta como "toda la población que desempeña cargos eclesiásticos, militares y administrativos, pues estos cargos durante la época estudiada suponía una rigurosa preparación y muchos estudios previos a la formación; por otro lado, la población que conforma la clase social baja es toda aquella que ejerce trabajos manuales y que se puede considerar que pertenece a los grupos socioeducacionales no privilegiados" (San Martín Gómez, s. f.).

20 Debido a que hasta el momento no existen estudios que traten esta cuestión con una metodología semejante, es imposible hacer la comparación con otras regiones de América. Por tanto, resulta interesante desarrollar acercamientos como estos para futuras investigaciones respecto a este tema en concreto.

21 En concreto, sobre esta cuestión solo existe hasta el momento el estudio de Boyd-Bowman (1970, p. 16), donde lleva a cabo la comparación de los nombres de criollos y castas de la Ciudad de México y Mérida durante el siglo XVII. 